\title{
Analysis of the potential correlation between gastric cancer and gastrointestinal microbiota via in-silico data mining
}

\section{Xuelu Ding}

Tianjin Medical University

\section{Yukun Zhu}

Tianjin Medical University

\section{Zhaoyuan She}

Tianjin Medical University

Xuewen Liu

Tianjin Medical University

Cancan Zhang

Tianjin First Central Hospital

\section{Xuying Ma}

Tianjin medical university

\section{Feng Wang}

Tianjin medical university

\section{Fei Wang}

Tianjin medical University

Xin Geng ( $\sim$ gengx@tmu.edu.cn )

Tianjin medical University https://orcid.org/0000-0003-0604-5392

\section{Research article}

Keywords: bioinformatics, microbiota, gastric cancer, databases

Posted Date: December 2nd, 2019

DOl: https://doi.org/10.21203/rs.2.17921/v1

License: (c) (i) This work is licensed under a Creative Commons Attribution 4.0 International License. Read Full License 


\section{Abstract}

Background: Emerging evidence shows the gastrointestinal microbiome might play an important role in the carcinogenesis of gastric cancer. While Helicobactor pylori has been reported to be a specific risk factor of gastric cancer, it is still controversial whether significant difference of non- $\mathrm{H}$. pylori microbiota exists between gastric cancer patients and healthy control.

Results: In this study, we employed multiple bioinformatic databases to excavate the potential correlation between gastrointestinal microbiome and gastric cancer. The databases involved in this investigation include HMDB, STITCH, OMIM, GWAS Catalog, WebGestalt, Toppgene, GeneMANIA. In addition, the network diagrams were built by use of Cytoscape software. Notably, our results showed that 33 common genes participate in both gastrointestinal microbiome and gastric cancer. The further analysis of these common genes suggested that there was a wide array of interactions and pathways in which the correlation between gastrointestinal microbiome and gastric cancer is involved.

Conclusions: Our present study gives a bioinformatic insight into possible pathways in which the gastrointestinal microbiome play roles in gastric cancer. Future efforts are necessary to be paid to elicit the exact mechanisms as well as potential therapeutic targets of gastric cancer.

\section{Introduction}

The gastric cancer (GC) accounts for 723,000 deaths annually in both sexes worldwide, being the third leading cause of cancer-related death. With devastating prognosis, the gastric cancer is becoming a heavy burden as well as a threat to human health (1).

The majority of microbes in our bodies reside in the gut with a profound influence on human physiology and pathology. The gene set of gut microbiota is 150 times larger than the human gene complement (2). The gastrointestinal ( $\mathrm{Gl}$ ) microbiota is crucial for the maintenance of human health, involving in energy metabolism (3), nutrient absorption (4) and the immune function (5). The change of the microbiota balance, which is called dysbiosis, contributes to the aberrant variation of microbiota diversity and abundance followed by the development of various disease, including gastrointestinal disease such as gastroesophageal reflux disease, gastritis and pseudomembranous colitis (6), metabolism disease such as obesity (7) and diabetes (8), cardiovascular disease(9), disorder of immunity(10), neuropsychiatric disease such as Parkinson disease, anxiety and autism spectrum disorder $(11,12)$, cancer-related disease such as hepatic carcinoma (13), colorectal carcinoma (14), pancreatic carcinoma $(15,16)$, breast cancer (17) and melanoma $(18,19)$.

The infection of Helicobacter pylori (H.pylori) is the strongest known risk factor for the development of gastric cancer $(20,21)$. Though very low proportion of the cases infected $H$. pylori progressed to gastric cancer (22), the eradication of $H$. pylori indeed contributes to a decrease of the incidence of gastric cancer (23). However, it is under controversial that whether the non-H.Pylori microbiota have a significant role in gastric cancer development. 
Dicksved et al. investigated patients with gastric cancer and dyspeptic controls. By use of the molecular profiling approach terminal restriction fragment length polymorphism and 16S rRNA gene cloning and sequencing, they found no significant difference between the gastric bacterial community from two groups. Sequencing of 140 clones revealed 102 phylotypes, with representatives from five bacterial phyla (Firmicutes, Bacteroidetes, Proteobacteria, Actinobacteria and Fusobacteria), while the abundance of H.pylori is relatively low (24).In non-H.pylori gastric cancer cases, Helicobacter cinaedi, Helicobacter mustelae and Campylobacter hyointestinalis were detected (25), while no significant role of these nonH.pylori bacteria in gastric cancer (26).

Some research suggest that a gradual shift of gastric microbiota community is likely to take place in the progression from precancerous lesions to gastrointestinal cancer, while different studies do not reach a consensus on the nature of the shift. Both increases $(27,28)$ and decreases $(29)$ in the bacterial diversity have been reported through comparing samples in each step of the gastric carcinogenesis, from chronic gastritis or intestinal metaplasia to gastric cancer. The increase in lachnospiraceae and members of the lactobaccillaceae and streptococcacaea family during the carcinogenesis were turned out to be the most consistent finding among the studies. Nevertheless, the exact role of these non-H.pylori bacteria in gastric carcinogenesis as well as the factors regulating the microbiota diversity are still remains to be determined. The discrepancy between the methods of different studies make it difficult to directly compare the results.

In this study, we employed multiple bioinformatics databases to further explore the possible association between gastrointestinal microbiota and gastric cancer in the level of interaction genes. Our data will help to provide several new insights for further investigation of the role of the gastrointestinal microbiota in the development of gastric cancer.

\section{Results}

\section{Analysis of the common genes shared by GI microbiota and GC}

\subsection{The identification of the genes related to GI microbiota}

10 metabolites of GI microbiota associated with gastric diseases were identified according to the HMDB database. These metabolites included Trimethylamine N-oxide, Butyric acid, Indoleacetic acid, Propionic acid, Indoxyl sulfate, p-Cresol, Acetone, Methane, 2-Methylerythritol and Glycine. 313 human genes associated with the 10 metabolites were identified by searching the STITCH database. The number of genes related to each metabolite is shown in Table 1.

\subsection{The identification of the genes related to GC}

464 genes from OMIM database and 34 genes from GWAS Catalog were shown to associated with GC. No overlap was found in genes obtained from the two databases.

\subsection{The common genes shared by GI microbiota and GC}


33 genes were identified to be the common genes shared by GI microbiota and GC. The symbol and description of each gene is shown in Table 2.

\section{Gene ontology analysis of the common genes shared by GI microbiota and GC}

Gene ontology analysis of 33 common genes shared by GI microbiota and GC conducted by WebGestalt is shown in Figure 2. The main biological process in which the 33 common genes enriched include cell communication, response to stimulus, biological regulation, multicellular organismal process, metabolic process, cellular component organization and location. The main molecular function of these genes include protein binding, hydrolase activity, nucleotide binding, ion binding, nucleic acid binding, transferase activity and molecular transducer activity. The main cellular components that these genes enriched in include membrane, macromolecular complex, vesicle, cell projection, vacuole, cytosol, nucleus, endomembrane system and cytoskeleton.

\section{Pathway analysis of the common genes shared by GI microbiota and GC}

The pathway analysis was conducted by WebGestalt and ToppGene. The pathway enrichment analysis results are shown in Supplementary Table 1. The pathways of the common genes shared by GI microbiota and GC involve G-protein activation, Opioid Signalling, Protein folding, Platelet activation, signaling and aggregation, ADP signalling through P2Y purinoceptor 12 , Regulation of insulin secretion, Morphine addiction, Retrograde endocannabinoid signaling, Glutamatergic synapse, Dopaminergic synapse, Apelin signaling pathway, Thromboxane signalling through TP receptor, ADP signalling through P2Y purinoceptor 1, Muscarinic acetylcholine receptor 2 and 4 signaling pathway, PI3K-Akt signaling pathway, Signal amplification, Cooperation of PDCL (PhLP1) and TRiC/CCT in G-protein beta folding, Thrombin signalling through proteinase activated receptors (PARs), Chaperonin-mediated protein folding, Prostacyclin signalling through prostacyclin receptor, $\mathrm{G}$ alpha (z) signalling events, $\mathrm{G}$ beta:gamma signalling through PLC beta, Adrenaline, noradrenaline inhibits insulin secretion, Presynaptic function of Kainate receptors, Glucagon-type ligand receptors, Opioid proopiomelanocortin pathway, Activation of Kainate Receptors upon glutamate binding, Opioid prodynorphin pathway, $\mathrm{Ca} 2+$ pathway, Enkephalin release, Cholinergic synapse, GABAergic synapse, Circadian entrainment, GABA B receptor activation, Activation of GABAB receptors, Glucagon-like Peptide-1 (GLP1) regulates insulin secretion, Cortocotropin releasing factor receptor signaling pathway, $5 \mathrm{HT} 1$ type receptor mediated signaling pathway, Vasopressin regulates renal water homeostasis via Aquaporins, Metabotropic glutamate receptor group II pathway, Opioid proenkephalin pathway, Integration of energy metabolism, Serotonergic synapse, G beta:gamma signalling through PI3Kgamma, Endogenous cannabinoid signaling, G alpha (12/13) signalling events, Heterotrimeric G-protein signaling pathway-rod outer segment phototransduction, G-protein beta:gamma signaling, Aquaporin-mediated transport, GABA receptor activation, Thyrotropin-releasing hormone receptor signaling pathway, Histamine $\mathrm{H} 1$ receptor mediated signaling pathway, $\mathrm{G}$ alpha (s) signalling events, Neurotransmitter Receptor Binding And Downstream Transmission In The Postsynaptic Cell, 5HT2 type receptor mediated signaling pathway, $\mathrm{G}$ alpha (q) signalling events, $\mathrm{G}$ alpha (i) signalling events, Glucagon signaling in metabolic regulation, Histamine $\mathrm{H} 2$ receptor mediated signaling pathway, GABA-B 
receptor II signaling, G protein gated Potassium channels, Inhibition of voltage gated $\mathrm{Ca} 2+$ channels via Gbeta/gamma subunits, Activation of $\mathrm{G}$ protein gated Potassium channels, Oxytocin receptor mediated signaling pathway, Beta3 adrenergic receptor signaling pathway, Alcoholism, Beta-catenin independent WNT signaling, $5 \mathrm{HT} 4$ type receptor mediated signaling pathway, Inwardly rectifying $\mathrm{K}+$ channels, Platelet homeostasis, Class B/2 (Secretin family receptors), Transmission across Chemical Synapses, Chemokine signaling pathway, Muscarinic acetylcholine receptor 1 and 3 signaling pathway, Heterotrimeric G-protein signaling pathway-Gq alpha and Go alpha mediated pathway, Pathways in cancer, Beta1 adrenergic receptor signaling pathway, Beta2 adrenergic receptor signaling pathway, GPCR downstream signaling, Signaling by GPCR, Signaling by Wnt, Neuronal System, Hemostasis, Gastrin-CREB signalling pathway via PKC and MAPK, PI3 kinase pathway, Angiotensin II-stimulated signaling through G proteins and betaarrestin, Ras signaling pathway, Metabotropic glutamate receptor group III pathway, Potassium Channels, Heterotrimeric G-protein signaling pathway-Gi alpha and Gs alpha mediated pathway, GPCR ligand binding, Dopamine receptor mediated signaling pathway, Wnt signaling pathway, Metabolism of proteins, Sphingosine 1-phosphate (S1P) pathway, Transmembrane transport of small molecules, S1P2 pathway, S1P3 pathway, Inflammation mediated by chemokine and cytokine signaling pathway, PLC beta mediated events, G-protein mediated events, S1P5 pathway, Alzheimer disease, S1P4 pathway, Long-term depression, Phototransduction, CXCR3-mediated signaling events, Thrombin signaling and proteaseactivated receptors, Activation of the phototransduction cascade, Bioactive Peptide Induced Signaling Pathway, Chagas disease (American trypanosomiasis), Inhibition of adenylate cyclase pathway, Adenylate cyclase inhibitory pathway, S1P1 pathway, Synthesis, secretion, and inactivation of Glucagonlike Peptide-1 (GLP-1), Aspirin Blocks Signaling Pathway Involved in Platelet Activation, Incretin synthesis, secretion, and inactivation, Visual signal transduction: Cones, Visual signal transduction: Rods, CXCR4 Signaling Pathway, Intracellular Signalling Through Prostacyclin Receptor and Prostacyclin, Vasopressin Regulation of Water Homeostasis, Intracellular Signalling Through PGD2 receptor and Prostaglandin D2, Phospholipids as signalling intermediaries, Nicotine pharmacodynamics pathway, Excitatory Neural Signalling Through 5-HTR 4 and Serotonin, Corticotropin Activation of Cortisol Production, PKC-catalyzed phosphorylation of inhibitory phosphoprotein of myosin phosphatase, How Progesterone Initiates Oocyte Membrane, Signaling Pathway from G-Protein Families, Inactivation, recovery and regulation of the phototransduction cascade, Excitatory Neural Signalling Through 5-HTR 7 and Serotonin, The phototransduction cascade, and Rapid glucocorticoid signaling.

\section{Analysis of interaction networks of the common genes shared by GI microbiota and GC}

The 33 common genes shared by GI microbiota and GC were further analyzed by GeneMANIA, and the interaction networks were mapped by Cytoscape software (Figure 3). Each node represents a particular gene. The color of the gene represents a kind of biological function. Here show the 7 main functions of the common genes, including heterotrimeric G-protein complex, extrinsic component of cytoplasmic side of plasma membrane, G-protein beta/gamma-subunit complex binding, extrinsic component of plasma membrane, extrinsic component of membrane, GTPase activity, response to glucagon. Each line of a color indicates a kind of interaction. The detail of the interactions is in Table 3. 


\section{Databases And Methods}

Multiple bioinformatic approaches were used to analyze the correlation between gastrointestinal microbiota and gastric cancer. All analyses were updated on May $31^{\text {st }}, 2018$. The databases and software adopted in this study contain HMDB, STITCH, OMIM, GWAS Catalog, WebGestalt, Toppgene, GeneMANIA and Cytoscape.

Since the enormous quantity of the species in human gastrointestinal tract and species vary across individuals, it is of great difficult to search genes related to gastrointestinal microbiota directly. We employed an indirect approach to take the metabolites of gastrointestinal microbiota as the intermediary. The genes associated with gastrointestinal microbiota metabolites were utilized as that related to gastrointestinal microbiota. The bioinformatic analytic procedures are shown in Figure 1.

HMDB, which is short for Human Metabolome Database (https://www.hmdb.ca), is a comprehensive internet resource focusing on metabolism and human metabolites and facilitating research on human metabolic science. Information in this database include the biological functions, physiological abundance, clinical connection, biochemical reaction, pathways of metabolism and reference spectra of complicated human metabolites(30).

STITCH, which is short for Search Tool for Interacting Chemicals (https://stitch.embl.de), mainly functions to integrate the interactions between small molecules and proteins. The database facilitates the users to obtain a view of the potential interaction network and effects of chemicals. The users are allowed to get the information on the binding affinities between proteins and chemicals and filter the tissue-specific proteins (31).

OMIM, which is short for Online Mendelian Inheritance in Man (http://omim.org), is a human Mendelian genetic-associated database mainly focusing on the human genes and phenotypes. The information of OMIM is based on published peer-reviewed biomedical literature and applied widely around in biomedical field(32).

GWAS Catalog, which is short for Genome-wide association studies Catalog (www.ebi.ac.uk/gwas), is an open-access collection of all the published genome-wide association studies (GWAS) and related results(33).

WebGestalt, which is short for WEB-based Gene SeT AnaLysis Toolkit (http://www.webgestalt.org), is a web tool mainly applied to conduct functional enrichment analysis, revealing the biological information of the high- throughput omics data (34).

ToppGene Suite (http://toppgene.cchmc.org) is a free and open access database providing information on gene list network enrichment, candidate gene prioritization and the identification and prioritization of candidate genes of novel disease (35). 
GeneMANIA (http://genemania.org) is a widely used web application to predict gene function, analyze gene lists and prioritize genes for functional assays. Based on the given query gene list, GeneMANIA search for the functionally similar genes, while a single query gene could generate genes with similar functions (36).

Cytoscape (www.cytoscape.org/) is designed to facilitate the visualization of molecular network and biological pathways. The software is able to depict large networks with more than ten thousand nodes and edges and display interactions between distinct molecular network constituents. The generation of biological networks are supported by the application of the editor module (37).

\section{Discussion}

The overlap of the genes related to GI microbiota and gastric cancer indicates that a probable correlation between GI microbiota and gastric cancer might exist. The further GO and pathway analysis suggest that the correlation is likely to be involved in widespread aspects which contain sophisticated interactions and mechanisms. The analysis of the 33 common genes will provide insight into further investigation into the mechanism of the gastric carcinogenesis and the potential diagnostic and therapeutic target of gastric cancer.

The main functions of the 33 common genes shared by GI microbiota and GC include GTPase activity, nucleoside-triphosphatase activity, pyrophosphatase activity, hydrolase activity, acting on acid anhydrides, in phosphorus-containing anhydrides, hydrolase activity, acting on acid anhydrides, G-protein beta/gamma-subunit complex binding, signal transducer activity, G-protein coupled receptor binding, Gprotein coupled serotonin receptor binding, GTP binding, guanyl ribonucleotide binding, guanyl nucleotide binding, signaling receptor binding, protein-containing complex binding, GTPase activating protein binding, purine ribonucleoside triphosphate binding, purine ribonucleoside binding, ribonucleoside binding, nucleoside binding, purine ribonucleotide binding, purine nucleotide binding and ribonucleotide binding.

The main pathways that 33 common genes enriched in mainly focus on $\mathrm{G}$ protein related pathways, several cell signal transduction pathways and synapse involving pathways. What is more, several rare pathways attracted our attention, such as opioid signaling and morphine addiction, the regulation of insulin secretion and inhibition as well as protein folding.

Opioids such as morphine have been taken advantage in analgesia for centuries. Data from 1980s drew attention on the effect on the growth of tumors. However, studies either in vivo or in vitro in order to elicit the effects of opioids shows conflicting results, making it still remains to be ascertained that whether the effects are beneficial or harmful. Though the analgesic effect of opioids is indispensable in clinical administration of tumor pain, opioids is also considered to be menacing for patients with tumor on account of the extra analgesia effects such as immune suppressive effects and angiogenic potential(38). Efforts have been paid to explore the correlation of gastric cancer and the administration of morphine. Xie et al. developed experiments to effects of the treatment of exogenous morphine on gastric cancer cell 
line MGC-803. The results showed that the treatment of morphine led to the significant inhibition of cell growth and proliferation as well as cell cycle arrest in the G2/M phase(39). Some other studies connected the morphine administration with gastric microbiota. It was reported that opioid induce gastric microbial disruption and bile dysregulation which further compromise the gastric barrier (40). Opioid is also reported to involve in the exacerbation of gram-positive sepsis which could be rescued by IL-17A neutralization (41). While the morphine and other narcotics have been noted to alter the composition of $\mathrm{GI}$ microbiota and promote the translocation of GI microbiota, it is reported that GI microbiota was indicated to play an essential role in modulating the response to chronic morphine administration(42). The opioids along with their effects on GI microbiota is also reported to have a role in the neuroinflammation in the central nervous system that is mediated by a gut-brain signaling axis, which further contributed to several inflammation-related psychopathologies. The difference in the opioid regimen significantly influence the GI microbiota as well as opioid dependence-related behaviors(43).

The endocannabinoid signaling is another pathway that the common genes significantly enriched in. In addition to be acknowledged to participate in the adipogenesis and obesity, the endocannabinoid system is currently under attention due to its potential therapeutic effects on a broad variety of disease including cancer(44). Drugs targeting the endocannabinoid system may have anti-metastatic effect to function against various types of tumors(45). Other functions of cannabinoid include anti-apoptotic, antiangiogenic and anti-proliferative in the cancer cell growth through multiple pathways such as ERK, Akt, MAPK, PI3K and HIF-1(46). The endocannabinoid signaling is also reported to play a role in inflammation(47), energy balance(47), gastric barrier (48), adipogenesis(48), and neuroimmune disorder(49) by modulating the gastric microbiota. However, no evidence showed the whether the crosstalk between endocannabinoid signaling and gastric microbiota involve in gastric cancer or not up to now.

Apelin is a member of the ligand of its receptor called APJ (50). Both apelin and APJ are generally expressed in blood vascular and reported to play a role in the angiogenesis by promoting the growth of endothelial cells (51-53). Additional studies demonstrated that apelin is related to the maturation of tumor blood capillaries(54), tumor growth and lymph node metastasis in vivo (55). Wang et al. developed a series of experiments to examine the role of apelin in gastric cancer. The results showed that the tumor apelin expression status was associated with the clinical features of gastric cancer including tumor differentiation, lymph node and distant metastases. The further in vitro studies revealed that apelin played a role in modulating the migration and invasion abilities of gastric cancer cell lines(56). There is also evidence supports that GI microbiota is specifically related to the regulation of the apelinergic system in the type 2 diabetes(57). However, it is still remains to be determined whether apelin participate in the effect of $\mathrm{Gl}$ microbiota on gastric carcinogenesis.

\section{Conclusions}

Our data suggest that the GI microbiota might take part in the development of gastric cancer. The correlation between GI microbiota and gastric cancer involves a wide array of functions, interactions and 
pathways. The results of our study provide bioinformatics insights into the potential correlation of $\mathrm{GI}$ microbiota and gastric cancer. The specific roles of particular pathway need further discussion and investigation by means of examination and analysis.

\section{Declarations}

\section{Ethics approval and consent to participate}

Not applicable.

\section{Consent for publication}

Not applicable.

\section{Availability of data and materials}

All data generated or analyzed during this study are included in this published article.

\section{Conflicts of Interest:}

No conflicts of interest declared.

\section{Funding:}

Research in the authors' lab is supported by Chinese National Natural Science Foundation Grant (No. 81671054 , No. $81771135 \otimes N o .31771520 \otimes N o .91649102)$, the key project of Tianjin Natural Science Grant (19JCZDJC35600).

\section{Authors' contributions:}

XG designed the study. XLD, YKZ, ZYS, XYM carried out the bioinformatic analysis. XLD, YKZ, XWL, CCZ wrote the manuscript. Feng W, Fei $W$ and $X G$ reviewed and revised the manuscript. All authors read and approved the final manuscript.

\section{Acknowledgements}

Not applicable.

\section{Abbreviations}

Gastric cancer: (GC)

Gastrointestinal: (GI)

Genome-wide association studies: (GWAS) 
Human metabolome database: (HMDB)

Online Mendelian Inheritance in Man: (OMIM)

Search Tool for Interacting Chemicals: (STITCH)

WEB-based Gene SeT AnaLysis Toolkit: (WebGestalt)

\section{References}

1.Ferlay J, Soerjomataram I, Dikshit R, Eser S, Mathers C, Rebelo M, et al. Cancer incidence and mortality worldwide: sources, methods and major patterns in GLOBOCAN 2012. International journal of cancer 2015,136:E359-86

2.Qin J, Li R, Raes J, Arumugam M, Burgdorf KS, Manichanh C, et al. A human gut microbial gene catalogue established by metagenomic sequencing. Nature 2010;464:59-65

3.Nieuwdorp M, Gilijamse PW, Pai N, Kaplan LM. Role of the microbiome in energy regulation and metabolism. Gastroenterology 2014,146:1525-33

4.Barratt MJ, Lebrilla C, Shapiro HY, Gordon JI. The Gut Microbiota, Food Science, and Human Nutrition: A Timely Marriage. Cell host \& microbe 2017,22:134-41

5.Belkaid Y, Harrison OJ. Homeostatic Immunity and the Microbiota. Immunity 2017;46:562-76

6. Kassam Z, Lee $\mathrm{CH}$, Yuan Y, Hunt RH. Fecal microbiota transplantation for Clostridium difficile infection: systematic review and meta-analysis. The American journal of gastroenterology 2013,108:500-8

7.Suarez-Zamorano N, Fabbiano S, Chevalier C, Stojanovic O, Colin DJ, Stevanovic A, et al. Microbiota depletion promotes browning of white adipose tissue and reduces obesity. Nature medicine 2015,21:1497-501

8.Forslund K, Hildebrand F, Nielsen T, Falony G, Le Chatelier E, Sunagawa S, et al. Disentangling type 2 diabetes and metformin treatment signatures in the human gut microbiota. Nature 2015,528:262-6

9.Tang WH, Kitai T, Hazen SL. Gut Microbiota in Cardiovascular Health and Disease. Circulation research 2017,120:1183-96

10.Kamada N, Seo SU, Chen GY, Nunez G. Role of the gut microbiota in immunity and inflammatory disease. Nature reviews Immunology 2013,13:321-35

11.Sharon G, Sampson TR, Geschwind DH, Mazmanian SK. The Central Nervous System and the Gut Microbiome. Cell 2016,167:915-32 
12.Hsiao EY, McBride SW, Hsien S, Sharon G, Hyde ER, McCue T, et al. Microbiota modulate behavioral and physiological abnormalities associated with neurodevelopmental disorders. Cell 2013,155:1451-63

13.Loo TM, Kamachi F, Watanabe Y, Yoshimoto S, Kanda H, Arai Y, et al. Gut Microbiota Promotes Obesity-Associated Liver Cancer through PGE2-Mediated Suppression of Antitumor Immunity. Cancer discovery 2017;7:522-38

14.Flemer B, Lynch DB, Brown JM, Jeffery IB, Ryan FJ, Claesson MJ, et al. Tumour-associated and nontumour-associated microbiota in colorectal cancer. Gut 2017,66:633-43

15.Quispe-Tintaya W, Chandra D, Jahangir A, Harris M, Casadevall A, Dadachova E, et al. Nontoxic radioactive Listeria(at) is a highly effective therapy against metastatic pancreatic cancer. Proceedings of the National Academy of Sciences of the United States of America 2013,110:8668-73

16.Fan X, Alekseyenko AV, Wu J, Peters BA. Human oral microbiome and prospective risk for pancreatic cancer: a population-based nested case-control study. 2018,67:120-7

17.Fuhrman BJ, Feigelson HS, Flores R, Gail MH, Xu X, Ravel J, et al. Associations of the fecal microbiome with urinary estrogens and estrogen metabolites in postmenopausal women. The Journal of clinical endocrinology and metabolism 2014,99:4632-40

18.Sivan A, Corrales L, Hubert N, Williams JB, Aquino-Michaels K, Earley ZM, et al. Commensal Bifidobacterium promotes antitumor immunity and facilitates anti-PD-L1 efficacy. Science 2015,350:1084-9

19.Gopalakrishnan V, Spencer CN. Gut microbiome modulates response to anti-PD-1 immunotherapy in melanoma patients. 2018,359:97-103

20.Bae SE, Choi KD. The effect of eradication of Helicobacter pylori on gastric cancer prevention in healthy asymptomatic populations. 2018,23:e12464

21.Suzuki $\mathrm{H}$, Matsuzaki J. Helicobacter pylori eradication failure may have confounded the recent largescale health database study that showed proton pump inhibitors increase gastric cancer risk. Gut 2018,67:2071-2

22. Uemura N, Okamoto S, Yamamoto S, Matsumura N, Yamaguchi S, Yamakido M, et al. Helicobacter pylori infection and the development of gastric cancer. The New England journal of medicine 2001,345:784-9

23.Ma JL, Zhang L, Brown LM, Li JY, Shen L, Pan KF, et al. Fifteen-year effects of Helicobacter pylori, garlic, and vitamin treatments on gastric cancer incidence and mortality. Journal of the National Cancer Institute 2012,104:488-92 
24.Dicksved J, Lindberg M, Rosenquist M, Enroth H, Jansson JK, Engstrand L. Molecular characterization of the stomach microbiota in patients with gastric cancer and in controls. Journal of medical microbiology 2009;58:509-16

25.Han HS, Lee KY, Lim SD, Kim WS, Hwang TS. Molecular identification of Helicobacter DNA in human gastric adenocarcinoma tissues using Helicobacter species-specific 16S rRNA PCR amplification and pyrosequencing analysis. Oncology letters 2010,1:555-8

26.Jo HJ, Kim J, Kim N, Park JH, Nam RH, Seok YJ, et al. Analysis of Gastric Microbiota by Pyrosequencing: Minor Role of Bacteria Other Than Helicobacter pylori in the Gastric Carcinogenesis. Helicobacter 2016,21:364-74

27.Eun CS, Kim BK, Han DS, Kim SY, Kim KM, Choi BY, et al. Differences in gastric mucosal microbiota profiling in patients with chronic gastritis, intestinal metaplasia, and gastric cancer using pyrosequencing methods. Helicobacter 2014,19:407-16

28.Wang L, Zhou J, Xin Y, Geng C, Tian Z, Yu X, et al. Bacterial overgrowth and diversification of microbiota in gastric cancer. European journal of gastroenterology \& hepatology 2016,28:261-6

29.Aviles-Jimenez F, Vazquez-Jimenez F, Medrano-Guzman R, Mantilla A, Torres J. Stomach microbiota composition varies between patients with non-atrophic gastritis and patients with intestinal type of gastric cancer. Scientific reports 2014;4:4202

30.Wishart DS, Feunang YD, Marcu A, Guo AC, Liang K, Vazquez-Fresno R, et al. HMDB 4.0: the human metabolome database for 2018. Nucleic acids research 2018;46:D608-d17

31.Szklarczyk D, Santos A, von Mering C, Jensen LJ, Bork P, Kuhn M. STITCH 5: augmenting proteinchemical interaction networks with tissue and affinity data. Nucleic acids research 2016;44:D380-4

32.Amberger JS, Bocchini CA, Schiettecatte F, Scott AF, Hamosh A. OMIM.org: Online Mendelian Inheritance in Man (OMIM(R)), an online catalog of human genes and genetic disorders. Nucleic acids research 2015;43:D789-98

33.MacArthur J, Bowler E, Cerezo M, Gil L, Hall P, Hastings E. The new NHGRI-EBI Catalog of published genome-wide association studies (GWAS Catalog). 2017;45:D896-d901

34.Wang J, Vasaikar S, Shi Z, Greer M, Zhang B. WebGestalt 2017: a more comprehensive, powerful, flexible and interactive gene set enrichment analysis toolkit. Nucleic acids research 2017;45:W130-w7

35.Chen J, Bardes EE, Aronow BJ, Jegga AG. ToppGene Suite for gene list enrichment analysis and candidate gene prioritization. Nucleic acids research 2009,37:W305-11

36.Franz M, Rodriguez H, Lopes C, Zuberi K, Montojo J, Bader GD, et al. GeneMANIA update 2018. Nucleic acids research 2018;46:W60-w4 
37.Kohl M, Wiese S, Warscheid B. Cytoscape: software for visualization and analysis of biological networks. Methods in molecular biology (Clifton, NJ) 2011,696:291-303

38.Brinkman D, Wang JH, Redmond HP. Morphine as a treatment of cancer-induced pain-is it safe? A review of in vivo studies and mechanisms. Naunyn-Schmiedeberg's archives of pharmacology 2018,391:1169-78

39.Qin Y, Chen J, Li L, Liao CJ, Liang YB, Guan EJ, et al. Exogenous morphine inhibits human gastric cancer MGC- 803 cell growth by cell cycle arrest and apoptosis induction. Asian Pacific journal of cancer prevention: APJCP 2012,13:1377-82

40.Banerjee S, Sindberg G, Wang F, Meng J, Sharma U, Zhang L, et al. Opioid-induced gut microbial disruption and bile dysregulation leads to gut barrier compromise and sustained systemic inflammation. Mucosal immunology 2016,9:1418-28

41.Meng J, Banerjee S, Li D, Sindberg GM, Wang F, Ma J, et al. Opioid Exacerbation of Gram-positive sepsis, induced by Gut Microbial Modulation, is Rescued by IL-17A Neutralization. Scientific reports 2015,5:10918

42.Kang M, Mischel RA, Bhave S, Komla E, Cho A, Huang C, et al. The effect of gut microbiome on tolerance to morphine mediated antinociception in mice. Scientific reports 2017;7:42658

43.Lee K, Vuong HE, Nusbaum DJ, Hsiao EY, Evans CJ, Taylor AMW. The gut microbiota mediates reward and sensory responses associated with regimen-selective morphine dependence.

Neuropsychopharmacology: official publication of the American College of Neuropsychopharmacology 2018

44.Schwarz R, Ramer R, Hinz B. Targeting the endocannabinoid system as a potential anticancer approach. Drug metabolism reviews 2018;50:26-53

45.Ramer R, Hinz B. Antitumorigenic targets of cannabinoids - current status and implications. Expert opinion on therapeutic targets 2016,20:1219-35

46.Javid FA, Phillips RM, Afshinjavid S, Verde R, Ligresti A. Cannabinoid pharmacology in cancer research: A new hope for cancer patients? European journal of pharmacology 2016;775:1-14

47.Cluny NL, Reimer RA, Sharkey KA. Cannabinoid signalling regulates inflammation and energy balance: the importance of the brain-gut axis. Brain, behavior, and immunity 2012,26:691-8

48.Cani PD. Crosstalk between the gut microbiota and the endocannabinoid system: impact on the gut barrier function and the adipose tissue. Clinical microbiology and infection: the official publication of the European Society of Clinical Microbiology and Infectious Diseases 2012,18 Suppl 4:50-3 
49.Mestre L, Carrillo-Salinas FJ, Mecha M, Feliu A, Guaza C. Gut microbiota, cannabinoid system and neuroimmune interactions: New perspectives in multiple sclerosis. Biochemical pharmacology 2018

50.He L, Xu J, Chen L, Li L. Apelin/APJ signaling in hypoxia-related diseases. Clinica chimica acta; international journal of clinical chemistry 2015;451:191-8

51.Kojima Y, Quertermous T. Apelin-APJ signaling in retinal angiogenesis. Arteriosclerosis, thrombosis, and vascular biology 2008,28:1687-8

52.Masri B, van den Berghe L, Sorli C, Knibiehler B, Audigier Y. [Apelin signalisation and vascular physiopathology]. Journal de la Societe de biologie 2009,203:171-9

53.Peltonen T, Napankangas J, Vuolteenaho O, Ohtonen P, Soini Y, Juvonen T, et al. Apelin and its receptor APJ in human aortic valve stenosis. The Journal of heart valve disease 2009;18:644-52

54.Kalin RE, Kretz MP, Meyer AM, Kispert A, Heppner FL, Brandli AW. Paracrine and autocrine mechanisms of apelin signaling govern embryonic and tumor angiogenesis. Developmental biology 2007,305:599614

55.Rayalam S, Della-Fera MA, Kasser T, Warren W, Baile CA. Emerging role of apelin as a therapeutic target in cancer: a patent review. Recent patents on anti-cancer drug discovery 2011,6:367-72

56.Feng M, Yao G, Yu H, Qing Y, Wang K. Tumor apelin, not serum apelin, is associated with the clinical features and prognosis of gastric cancer. BMC cancer 2016,16:794

57.Geurts L, Lazarevic V, Derrien M, Everard A, Van Roye M, Knauf C, et al. Altered Gut Microbiota and Endocannabinoid System Tone in Obese and Diabetic Leptin-Resistant Mice: Impact on Apelin Regulation in Adipose Tissue. Frontiers in Microbiology 2011,2

\section{Tables}

Table 1. The metabolites of GI microbiota and associated gene number

\begin{tabular}{ccc}
\hline HMDB ID & Name & Genes \\
\hline HMDB0000925 & Trimethylamine N-oxide & 36 \\
HMDB0000039 & Butyric acid & 54 \\
\hline HMDB0000197 & Indoleacetic acid & 57 \\
\hline HMDB0000237 & Propionic acid & 59 \\
\hline HMDB0000682 & Indoxyl sulfate & 27 \\
HMDB0001858 & p-Cresol & 25 \\
\hline HMDB0001659 & Acetone & 51 \\
HMDB0002714 & Methane & 23 \\
\hline HMDB0011659 & 2-Methylerythritol & 9 \\
HMDB0000123 & Glycine & 49 \\
\hline
\end{tabular}




\begin{tabular}{|c|c|}
\hline Gene & Full name of gene \\
\hline $\mathrm{TRH}$ & thyrotropin releasing hormone \\
\hline GNA11 & G protein subunit alpha 11 \\
\hline GNA12 & G protein subunit alpha 12 \\
\hline GNA14 & G protein subunit alpha 14 \\
\hline MTRNR2L2 & MT-RNR2 like 2 \\
\hline GNAI1 & G protein subunit alpha i1 \\
\hline GNAI3 & G protein subunit alpha i3 \\
\hline GNAO1 & G protein subunit alpha o1 \\
\hline MTOR & mechanistic target of rapamycin kinase \\
\hline GNAT1 & G protein subunit alpha transducin 1 \\
\hline GNAT2 & G protein subunit alpha transducin 2 \\
\hline GNAT3 & G protein subunit alpha transducin 3 \\
\hline BCAS2 & pre-mRNA-splicing factor \\
\hline GNB1 & G protein subunit beta 1 \\
\hline GNB2 & G protein subunit beta 2 \\
\hline GNB2L1 & guanine nucleotide-binding protein subunit beta-2-like 1 \\
\hline GNB3 & G protein subunit beta 3 \\
\hline GNB4 & G protein subunit beta 4 \\
\hline TRIO & trio Rho guanine nucleotide exchange factor \\
\hline GNG2 & G protein subunit gamma 2 \\
\hline GNG3 & G protein subunit gamma 3 \\
\hline GNG4 & G protein subunit gamma 4 \\
\hline GNG5 & G protein subunit gamma 5 \\
\hline GNG7 & G protein subunit gamma 7 \\
\hline TRIT1 & tRNAisopentenyltransferase 1 \\
\hline GNGT1 & G protein subunit gamma transducin 1 \\
\hline GNGT2 & G protein subunit gamma transducin 2 \\
\hline RSPO1 & R-spondin 1 \\
\hline GNG10 & G protein subunit gamma 10 \\
\hline GNG11 & G protein subunit gamma 11 \\
\hline GNG12 & G protein subunit gamma 12 \\
\hline GNG13 & G protein subunit gamma 13 \\
\hline PCSK9 & proprotein convertase subtilisin/kexin type 9 \\
\hline
\end{tabular}


Table 3. The interaction patterns and their proportions of protein

\begin{tabular}{cc}
\hline Network Categories & Percentage \\
\hline Shared protein domains & $45.34 \%$ \\
Physical Interactions & $34.19 \%$ \\
Predicted & $12.40 \%$ \\
Pathway & $6.50 \%$ \\
Co-expression & $1.21 \%$ \\
Co-localization & $0.36 \%$ \\
\hline
\end{tabular}

Figures 


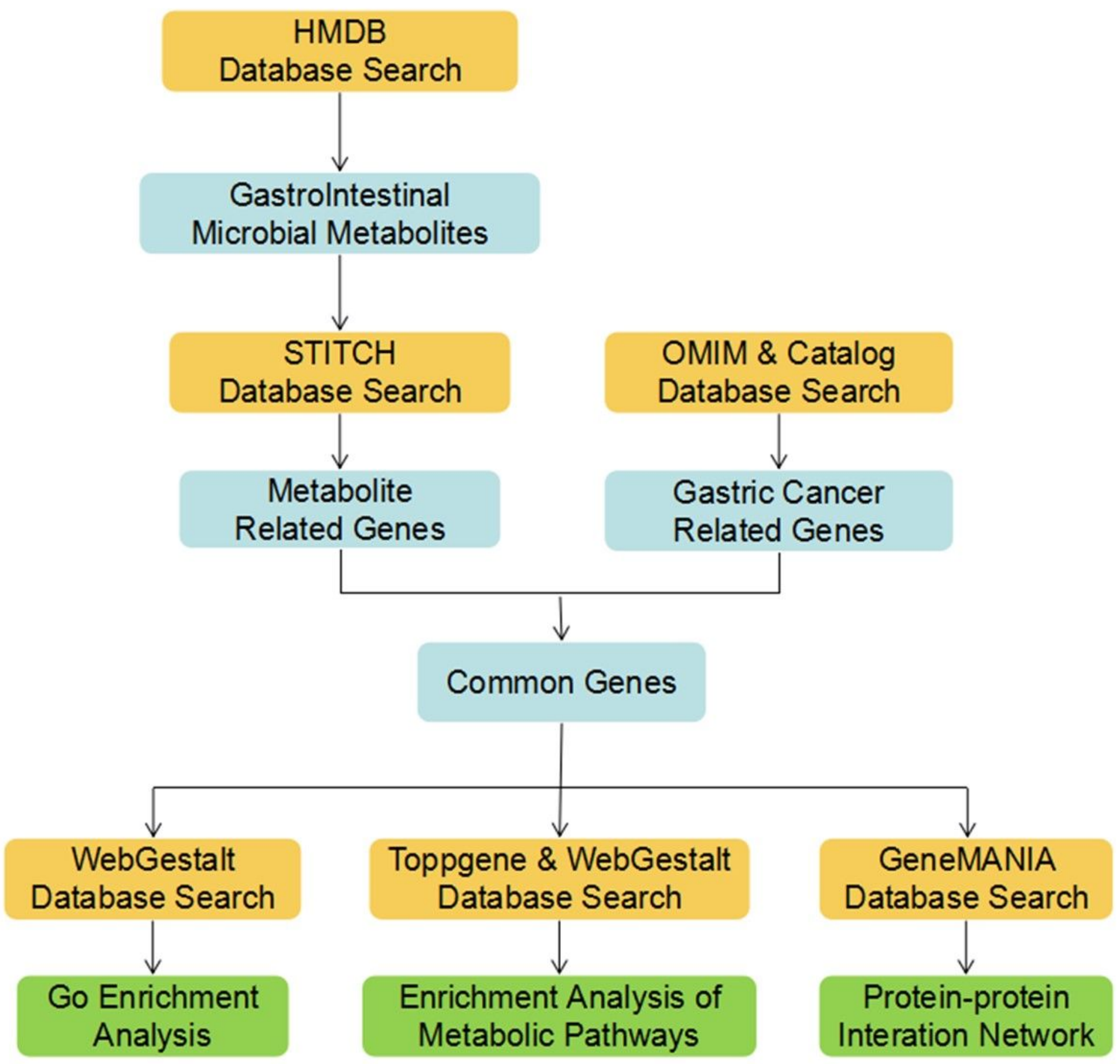

Figure 1

Multiple bioinformatic approaches were used to analyze the potential interaction between gastrointestinal microbiota and gastric cancer. The bioinformatic analytic procedures are shown. 
Bar chart of Biological Process categories

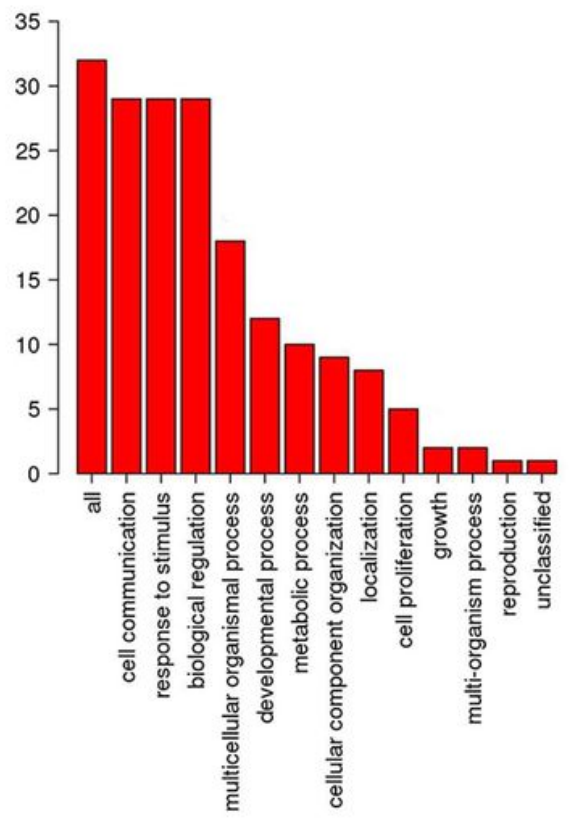

Bar chart of Cellular Component categories

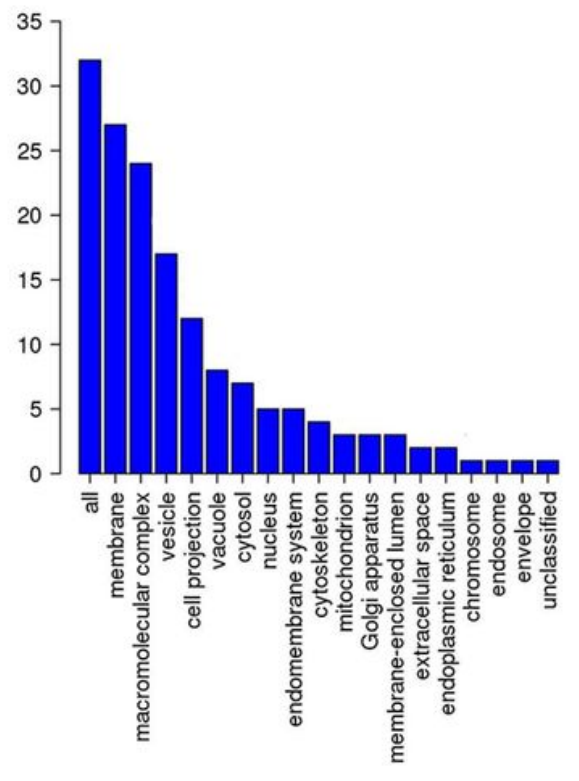

Bar chart of Molecular Function categories

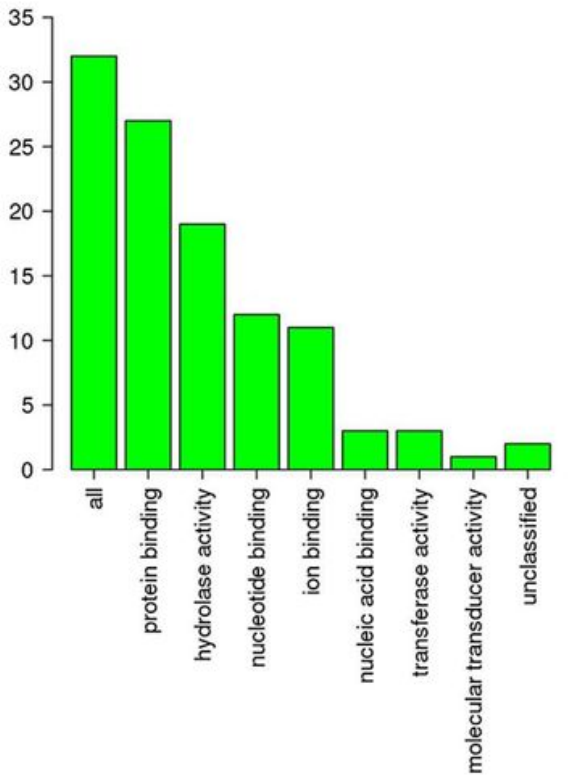

Figure 2

Gene ontology analysis of 33 common genes shared by GI microbiota and GC conducted by WebGestalt is shown. 


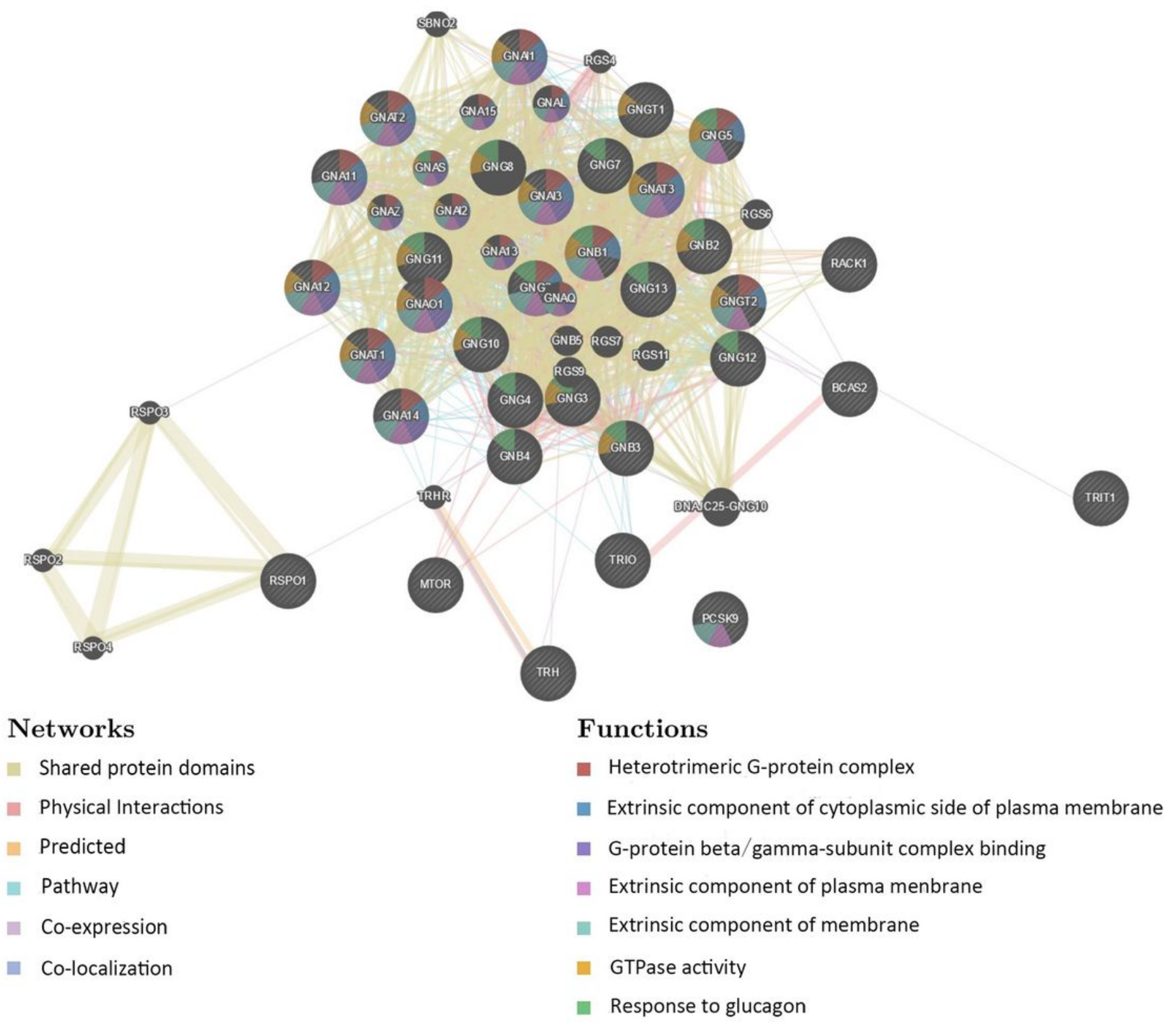

Figure 3

The 33 common genes shared by GI microbiota and GC were further analyzed by GeneMANIA, and the interaction networks were mapped by Cytoscape software.

\section{Supplementary Files}

This is a list of supplementary files associated with this preprint. Click to download.

- SupplementaryTable1.Pathwayanalysis.doc 